\title{
CONFIGURATIONAL SPLITTING OF ELECTRON RADIATION ON ATOMS AND ITS DISCOVERY ON ACCELERATORS
}

\author{
V.Grishin*, S.Likhachev, G.Nefedov, Nuclear Physics Institute of Moscow State University \\ 119899 Moscow, Russia
}

\begin{abstract}
New effects in coherent polarization bremsstrahlung radiation (PB) of relativistic electrons on atoms of light elements with the clearly expressed atomic shell structures are indicated. Here coherent PB acquires the special structure split on two low and high frequency regions that is most noticeable in media with atomic numbers $Z<10$. As a whole coherent PB spectrum displaces in low frequency region contrasting to earlier known predictions. It is manifested that there are real possibilities of splitting discovery on electronic accelerators. Special computer code designed for empiric data processing is discussed.
\end{abstract}

\section{INTRODUCTION}

The polarization bremsstrahlung radiation $(\mathrm{PB})$ is a result of scattering of electromagnetic field of a fast charged particle on atomic electrons [1] This is one of the new interesting problems of charge particle radiation physics. In the region of energies of $\mathrm{X}$-ray photons up to tens $\mathrm{keV}$, most interested to the different applications, PB acquires a collective character because the process of a dispersion "comprises" atomic electrons coherently .

As result the PB properties become highly sensitive for matter structure that was proved experimentally in particular in Ref.[2,3]. This confirms once more perspectives of PB utilization for development of new methods of matter diagnostics and other applications.

Nevertheless despite of successes in PB study, influence of atom shell structure on collective properties PB is insufficiently studied up to now (usually shell effects are being considered only at photon energies of about tens - hundreds $\mathrm{eV}$, by reference for intratomic transitions [4]). Meanwhile in light elements the electron distribution has well distinct shell structure especially in the considered below region of atomic number $Z<10-15$. The electron distributions, obtained in Ref.[5] by Hartree-Fok's method calculation, demonstrate that the differences in spatial locations of electronic shells are very noticeable. Here there is the sharp K-shell peaks displaced to atomic center and occupied by two electrons. But $(Z-2)$ electrons, forming in fact combined external shells, are displaced rather considerably to the atomic peripheries. In aggregate a average atomic radius of light elements reaches to order of $1-2$ atomic units. At the same time characteristic radius magnitude of electron distribution according to Thomas-Fermi's

\footnotetext{
* grishin@depni.npi.msu.su
}

model [6] makes on an order of $1 / Z^{1 / 3} \simeq 0.5-0.7$ atomic units.

As it is shown by authors in Ref.[5] (in a case only of low-relativistic electrons), two effects are found out. The PB collective process (i.e. coherence) in light atoms will be exhibited as a whole in more long-wave region.

Then due to noticeable difference in an allocation of electronic shells, the region of collective processes will be split on two ones. The last phenomenon was termed as configuration splitting (by analogy with known effect in shell photonuclear interactions [7]).

The propose of this report is to extend our estimations on more interesting case of relativistic particles and to analyze possibilities of effects detection in a real experiment.

\section{THEORETICAL ESTIMATION}

The electromagnetic field of a fast electron moving in a direction $z$ with velocity $v$ and impact parameter $b$ by atom, can be represented as a wave package termed frequently as the package of virtual (or equivalent) photons $[8,9]$ :

$$
E(t, z)=\int_{-\infty}^{\infty} E_{\omega} \exp (-i \omega(t-z / v)) d \omega
$$

$E_{\omega}=e \zeta K_{1}(\zeta) /(\pi b v), \zeta=(\omega b) /(\gamma v), K_{1}$ is modified Hankel's function, $e$ and $\gamma$ are charge and relativistic factor of an electron. The quantity $E_{\omega}$ remains approximately constant up to $\zeta \simeq 1$, and then wanes sharply. The wave vectors in the package $\mathbf{k}_{\omega}=\mathbf{n} \omega / v$; the unit vector $\mathbf{n}$ is directed along the axis $z$.

As was mentioned the mechanism PB can be presented as a dispersion of virtual photons on atomic electrons. Basing on that dispersed photon energy exceeds a binding energy of atomic electrons essentially, one can represent this process as a sort of collective Compton's scattering. Utilizing known procedure $[9,10]$, we obtain the spectral-angular PB intensity of energy radiated of an incident charge particle per unit length of its pass in a medium with density $n_{o}$ along a unit vector $\mathbf{n}^{\prime}$ under at angle $\psi$ with respect to the axis $z$ (after performing the integration on acceptable values of impact parameter $b$, then also averaging on mutual orientations of incident fast particle and atoms and on distribution of atomic electrons )

$$
\frac{d^{3} W}{d \omega d \Omega d z}=C Z_{e f f}^{2} L_{\omega} Y_{\psi}
$$


where

$$
\begin{gathered}
Z_{e f f}^{2}=<\left|\sum_{s=1}^{Z} \exp \left(-i \mathbf{q}_{\omega} \mathbf{r}_{\mathbf{s}}\right)\right|^{2}>, \\
L_{\omega}=\ln \left(\gamma v / \omega R_{\omega}\right)+f, \\
Y_{\psi}=\left(1+\cos ^{2} \psi\right) / D^{2}+\varepsilon^{2}(1-\cos \psi)^{2} / D^{3}, \\
D=1+\varepsilon(1-\cos \psi), \varepsilon=, \hbar \omega / m c^{2}
\end{gathered}
$$

Here $d \Omega$ is an element of a solid angle , $C=$ $\left(e^{6} n_{o}\right) /\left(2 \pi m^{2} c^{3} v^{2}\right)$, vector $\mathbf{q}_{\omega}=\mathbf{k}_{\omega}-\mathbf{k}_{\omega}, q_{\omega} \simeq$ $2(\omega / c) \sin (\psi / 2)$ as $v \sim c, \mathbf{k}$ ' is a wave vector of a wave dispersed along $\mathbf{n}$, and $\mathbf{r}_{\mathbf{s}}$ are radius-vectors of atomic electrons. Parameter $R_{\omega}$ coinciding with a minimum of impact parameter is accepted equal to average radius of atomic shells [1], $f$ is a small term which should be taken in account in the region of maximum frequencies (see below). Factor $Z_{\text {eff }}^{2}$ reflects paired correlations between atomic electrons averaged on shell distribution. Thus factor $Z_{e f f}^{2}$ is a coherent parameter (or atomic formfactor) of BP process. This procedure of an average gives

$$
Z_{e f f}^{2}=Z+\frac{(Z-1)}{Z}\left[\int_{0}^{\infty}\left(\sin \left(q_{\omega} r\right) / q_{\omega}\right) \rho r d r\right]^{2} .
$$

where $\rho=\rho(r)$ is spatial average density of atomic electron distribution ( below it is calculated by Hartree-Fok's method).

\section{DISCUSSION AND PREDICTION}

The expressions received above allow establish an influence of relativistic energy of incident particles on PB property. With $\gamma$ growth, the form of a virtual photon spectrum does not change but limiting frequency of spectrum is increased. Therefore the magnitude of coherence parameter changes only in high-frequency area. But the PB intensity integrated on all spectrum grows approximately proportionally to $\gamma$.

In a result we have for $Z_{\text {eff }}^{2}$ the picture with very characteristic peculiarities mentioned above, see fig. 1 and 2 where some data of digital calculations are presented.

Two regions of coherent radiation clearly are observed. Firstly two electrons of the inferior shell are coherently enveloped that is happening in case of carbon atoms, in correspondence to value of its medial radius, already at energies of radiated photons about $30 \mathrm{keV}$ at $\psi=\pi / 4$, and less than $20 \mathrm{keV}$ at $\psi=\pi / 2$. At the same time electrons of the upper shells experience a coherent correlations since energy about $10 \mathrm{keV}$, and the noticeable total coherence is watched only at energy less than $5 \mathrm{keV}$.

The PB angular distribution keeps dipole character in the basic zone of coherence radiation and only in high frequency of area PB becomes obvious not isotropic. So at electron energies 10,100 and $1000 \mathrm{MeV}$ we have $\hbar \omega_{\max } \simeq 40,400$, and $4000 \mathrm{keV}$. The ratio $Y(\psi=$ $\pi) / Y(\psi=0) \simeq 1,0.44$, and 0.03 respectively.

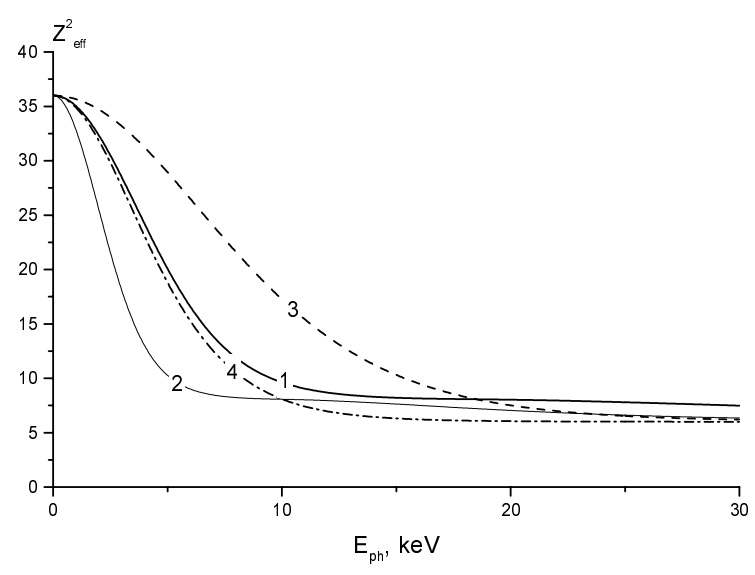

Figure 1: Coherence parameter $\left\langle Z_{\text {eff }}^{2}>\right.$ as function of PB photon energy in carbon ( $Z=6$ ) for shell model (solid curves 1,2) and traditional screen model (dashed curves 3,4 ). Curves 1,3 at $\psi=\pi / 4$, curves 2,4 at $\psi=\pi / 2$.

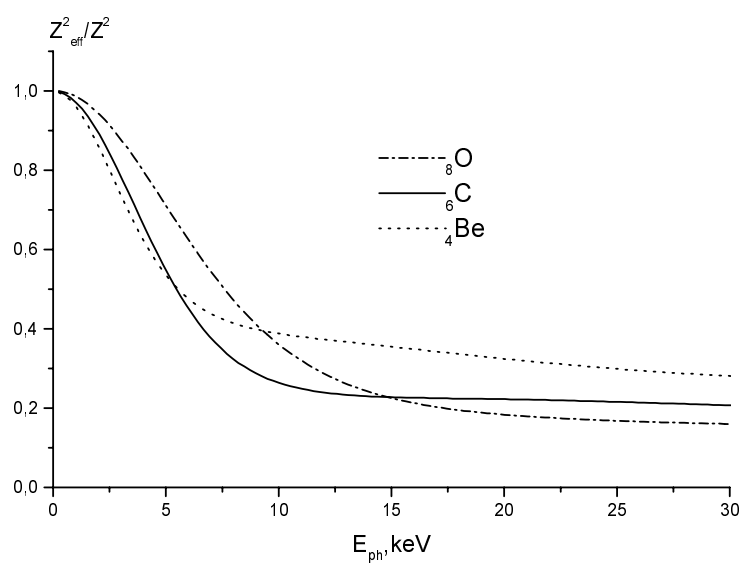

Figure 2: Reduced coherence parameter $<Z_{\text {eff }}^{2} / Z^{2}>$ as function of PB photon energy in different light atoms at $\psi=\pi / 4$.

The results obtained can be utilized for more precise description of fast electron radiation properties in both amorphous and structured matters. So, in ref. [3] PB properties in dense amorphous carbon were explored. Formally the estimates obtained above are applicable only for rarefied mediums. However the most important in amorphous mediums effect of density is exhibited only for photons of low energy in the region of $1-3 k e V$. Therefore the marked in ref. [3] displacement of coherent processes to a region of energy of coherent processes to a region of energies $<5 \mathrm{keV}$ can be bound in some respect with shell effects.

The experimental detection of the marked effects in many respects is defined by a choice of observation con- 
ditions. As it is followed from data of fig.1, the most interesting region of measurement is lying in the interval of photon energy from some $\mathrm{keV}$ to $20-40 \mathrm{keV}$.

First of all let to take into account that due to the factor $L_{\omega}$, the splitting effect is smoothed in real PB spectrum . Besides it is necessary to estimate attendant and background events. The conventional bremsstrahlung radiation is the most intensive attendant radiation. But the latter is focused in an angle interval $\Delta \psi \simeq 1 / \gamma$ ), and PB becomes dominant for major angles.

Apparently most strong deformations arise in process of PB detection (nonuniform spectral sensitivity and limited energy resolution of instrumentation). For elimination of similar deformations the special iterative code is elaborated that permits to restore PB spectrum, passing the traditional procedure with using of an inverse matrix. Model testing has shown, that at "experimental" measuring with statistical errors about several percents, the reliable restitution of "latent" structure of spectrums( including coherence parameter structure) is possible, see fig. 3 .

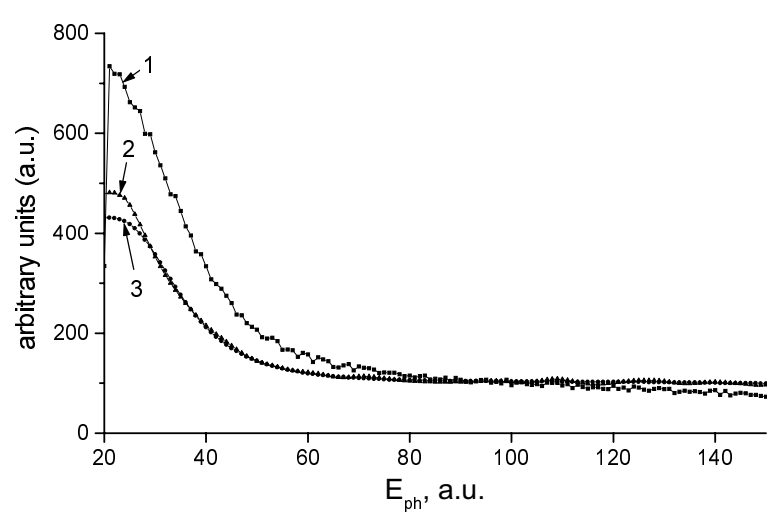

Figure 3: "Experimental" PB spectrum (curve 1), theoretical coherent parameter (curve 2) and coherent parameter restored (curve 3 ) as functions of photon energy (in arbitrary units; the initial point on photon energy axis coincide with a left-hand boundary of detector sensibility at about 1 keV. )

Here in photon energy area, most important for an analysis of splitting effect, the theoretical and restored curves are coincided rather satisfactory. The plateau of energy region, where the values of coherence parameter are determined by the sum of two coherent $\mathrm{K}$ - shell electrons and incoherent $Z-2$ ones, is clearly allocated. Restoring process "works" very stable in condition of statistical providing in real experimental measurements. Therefore it is possible to realize an effective study of PB applications on modern electron accelerators.

The authors are very thankful to N.M.Kabachnik for the submitted program of atomic shell caiculations and to B.M.Bolotovsky, B.S.Ishkhanov and N.N.Nasonov for helpful discussions on the obtained results.

The work is carried out under support of Russian Foundation for Basic Researches, grant No. $00-02-17734$.

\section{REFERENCES}

[1] M. Amus'ia, V.Buimistrov, B.Zon et al., Polarization Bremsstrahlung of particles and atoms, Plenum Press, N.Y. 1992.

[2] S.Blazhevich, A.Chepurnov, V.Grishin et al. // Physics Letters A 254 (1999) 203-232.

[3] S.Blazhevich, A.Chepurnov, V.Grishin et al. // Phys. Lett. A 211 (1996) 309.

[4] A.V.Korol, A.V.Solov'ev // J.Phys. B, 30 (1997) 1105.

[5] V.Grishin, S.Likhachev//Physics Letters A 282/3 (2001).

[6] L.D.Landau, E.M.Lifshits, Quantum mechanics, $\S 70$.

[7] R.A.Eramzhan, B.S.Ishkhanov, I.M.Kapinonov and V.S.Neudachin//Physics Reports, v.136, n 4-6, 1986.

[8] W.Panofsky, M.Phillips, Classic Electricity and Magnetism, Adison-Wesley Publishing Company Inc, Cambridge 42, Mass, $\S 18.5$

[9] A.I.Ahiezer, V.B.Berestetsky, Quantum electrodynamics, Moscow,Nauka.

[10] L.D.Landau, E.M.Lifshits, Field theory, Moscow, 1960, $\S 80$. 